(C) 2018 IEEE. Personal use of this material is permitted. Permission from IEEE must be obtained for all other uses, in any current or future media, including reprinting/republishing this material for advertising or promotional purposes,creating new collective works, for resale or redistribution to servers or lists, or reuse of any copyrighted component of this work in other works.

\title{
Migration Planning from Elastic Optical Networks to Spectrally-Spatially Flexible Optical Networks
}

\author{
Piotr Lechowicz \\ Department of Systems and Computer \\ Networks, Faculty of Electronics \\ Wroclaw University. of Science and \\ Technology \\ Wroclaw, Poland \\ Piotr.Lechowicz@pwr.edu.pl \\ Salvatore Spadaro \\ Universitat Politècnica de Catalunya \\ (UPC) \\ Barcelona, Spain
}

\author{
Rubén Rumipamba-Zambrano \\ Universitat Politècnica de Catalunya \\ (UPC) \\ Barcelona, Spain
}

Jordi Perelló

Universitat Politècnica de Catalunya (UPC)

Barcelona, Spain

\author{
Krzysztof Walkowiak \\ Department of Systems and Computer \\ Networks, Faculty of Electronics \\ Wroclaw University. of Science and \\ Technology \\ Wroclaw, Poland
}

\begin{abstract}
We focus on a migration planning from elastic to spectrally-spatially flexible optical networks by upgrading regular with SDM-capable ROADMs. Our most promising strategy reveals that by migrating $30 \%$ of the regular ROADMs a $2 x$ carried traffic increase can be achieved.
\end{abstract}

Keywords-spectrally-spatially flexible optical networks, migration planning, elastic optical networks

\section{INTRODUCTION}

The continuous growth of the overall Internet traffic is leading to an exhaustion of the available spectral resources. Possible solutions to overcome this problem are spectrallyspatially flexible optical networks (SS-FONs). SS-FONs combine two network technologies, namely, flex-grid (elastic) optical networks (EON) and spatial division multiplexing (SDM), yielding an enormous capacity increment [1]. Moreover, SS-FONs allow for an adaptive use of different modulation formats (MFs), multi-carrier (i.e., super-channel, SCh) transmission, flexibility in resource management due to the utilization of the new spatial dimension and potential resource savings through integrated network devices. The core idea of SS-FONs is to enable a parallel transmission of several co-propagated spatial channels in suitably designed optical fibers, such as multicore fibers, multi/few-mode fibers or single-mode fiber bundles (SMFBs), which aggregate many single-core and single-mode fibers (SMFs) in a fiber bundle [2]. The key components required to route the traffic in the network are the reconfigurable optical add-drop multiplexers (ROADMs), which are also expected to be SDM-capable in the future (SDM-ROADMs). One of the possible SDMROADMs assumed in the literature are fully non-blocking broadcast-and-select ROADMs supporting lane changes (FNB SDM-ROADMs) [3].

Although EON and SDM are orthogonal technologies in principle, their expected future combination in SS-FONs is an interesting case of study in network planning scenarios (not addressed in detail in the literature, to the best of our knowledge). This work aims to study the gradual migration from EONs to SS-FONs from a network node perspective, considering that all links are equipped with SMFBs (the near-term SDM solution) since the network operators will likely seek to leverage, as starting point, their already deployed current infrastructure [4]. In addition, this type of fibers allows isolating the impact of crosstalk from the study, while compatible with FNB SDM-ROADMs. We evaluate the performance of several strategies to upgrade regular ROADMs for SMFs to SDM-ROADMs in order to support forecast traffic scenarios.

\section{NETWORK MODEL AND PROPOSED SOLUTION}

We assume that the network deploys SMFBs, where each bundle contains C SMFs. Each node is equipped either with regular ROADMs for SMFs or B\&S FNB SDM-ROADM (see Fig. 1). The assumed SDM-ROADM is capable to direct any input fiber to any output fiber, i.e., it supports lane changes [3]. Transmission using more than one fiber in the SMFB is possible only when both connected nodes are SDM-capable. Otherwise, only one fiber of the SMFB can be utilized (as in EONs over SMFs).

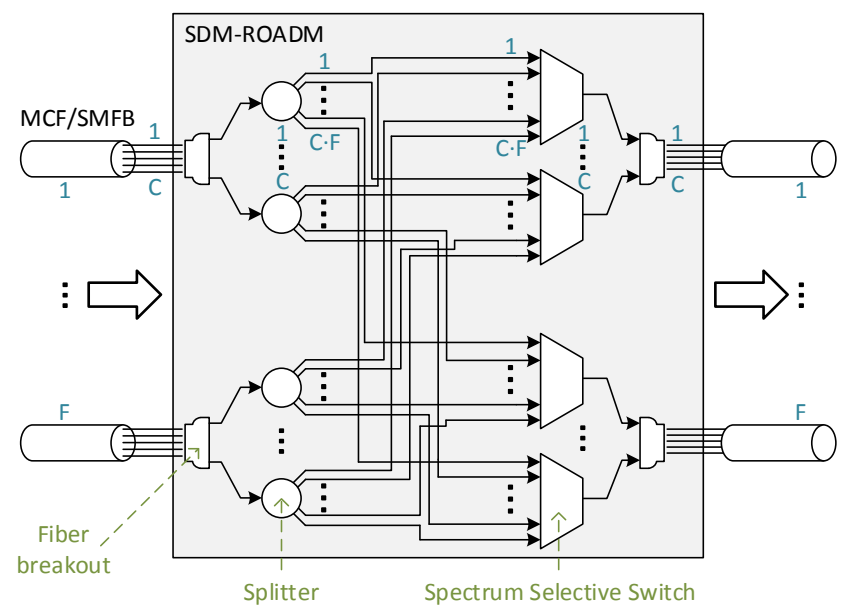

Fig. 1. B\&S FNB SDM-ROADM.

In this scenario, when all forecast traffic will not be allocated in an EON over SMFs solely, the need for enabling other deployed dark fibers arises. Nevertheless, one critical decision about this concern is which network node(s) (and therefore, network links) have to be upgraded in order to support SDM. To this aim, we propose several strategies for node selection: i) a reference one, which is the node with the highest degree (ND); ii) the node carrying the highest value of subcarriers (NCS); iii) the node carrying the highest value of bit-rate (NCB); iv) the ending nodes of the link carrying 
the highest value of subcarriers (LCS); v) the ending nodes of the link carrying the highest value of bit-rate (LCB). Nodes are selected and upgraded one-by-one according to the applied strategy, until the whole traffic can be allocated in the network, i.e., at each step only one node is selected and if the number of spatially enabled fibers in the network increases, the traffic is reallocated. Note that, at each step, only those nodes still not upgraded are considered for selection.

In order to allocate the offered demands in the network, the k-shortest path first-fit method is used. At first, demands are sorted in descending order, according to the required number of subcarriers on their shortest path. Then, demands are processed consecutively, and for each one, all candidate paths are checked, and the request is provisioned using the lowest optical corridor found, i.e., the highest allocated slice in the network is minimized.

\section{NUMERICAL RESULTS}

We evaluate our proposed solution in a national US network topology composed of 26 nodes and 84 links, with an average link length of $755 \mathrm{~km}$. The considered SS-FON is composed of SMFBs, where each fiber provides $4 \mathrm{THz}$ bandwidth divided into frequency slices of $12.5 \mathrm{GHz}$ width. We assume that the network is equipped with coherent transceivers, each one operating at a fixed baud rate and transmitting/receiving an optical carrier (OC) that occupies $37.5 \mathrm{GHz}$ (3 frequency slices) [5]. We consider 4 available MFs, namely, BPSK, QPSK, 8-QAM,16-QAM that support bit-rates of 50, 100, 150 and $200 \mathrm{Gbps}$, since a fixed guardband of $12.5 \mathrm{GHz}$ width is assumed to separate adjacent SChs. Moreover, transmission reaches for each of the considered MFs are assumed to be 6300, 3500, 1200 and 600 $\mathrm{km}$, respectively [4]. If the requested bit-rate exceeds a single transceiver capacity for the applied MF, the request is transmitted over several OCs within one SCh using a set of adjacent slices (i.e., configuring a spectral $\mathrm{SCh}$ ).

In most of the experiments, we assume that the number of fibers per SMFB is $\mathrm{C}=7$ and the number of candidate (shortest) routing paths for each demand is $\mathrm{k}=10$. For each path, we select the MF with the highest spectral efficiency supporting the required transmission reach. The overall offered traffic volume is between 60 and 200 Tbps, with a 20 Tbps granularity, and for each value, we evaluate 10 randomly generated sets of demands. Each demand has randomly selected source/destination nodes and a bit-rate uniformly selected within the range from 50 Gbps to $1 \mathrm{Tbps}$, with 50 Gbps granularity. As the main performance metric, we take the ratio of upgraded nodes to all nodes (node migration ratio, abbr. NMR) that allows for pending traffic allocation. Moreover, we also record the ratio of spatially enabled links to all links in the network (fiber migration ratio, abbr. FMR).

In Fig. 2, we present NMR as a function of the total offered traffic for different node selection strategies. As we can see, LCS outperforms other strategies, especially for medium values of traffic load. In particular, by migrating $30 \%$ of regular ROADMs, a 2 times carried traffic increase can be achieved. It is natural, that for large traffic volumes, NMR converges to the same value, as most of the nodes are upgraded, and the order in which they are selected is irrelevant. In Fig. 3, we report FMR as a function of total offered traffic volume for different node selection strategies.
Trends are similar to those presented in Fig. 2. As LCS provides the best results, it is used in further experiments.

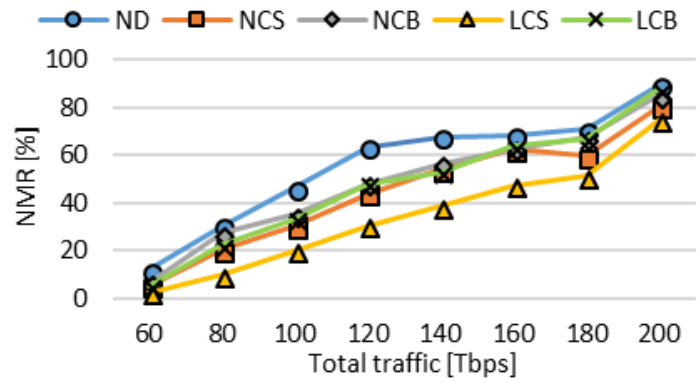

Fig. 2. Node migration ratio as a function of the total traffic.

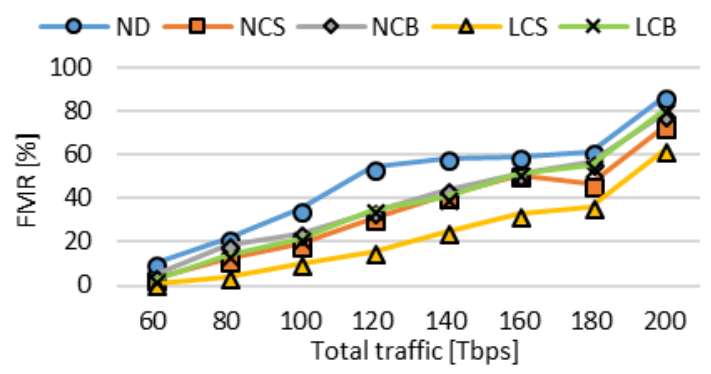

Fig. 3. Fiber migration ratio as a function of the total traffic.

Fig. 4 presents NMR and FMR as a function of the number of candidate paths for a total offered traffic volume of 140 Tbps. $S_{e}$ and $S_{f}$ denote the average highest allocated slice index among all links and average highest allocated slice index among all fibers, respectively. As can be observed, NMR and FMR decrease about $37 \%$ and $50 \%$, respectively, between 1 and 5 candidate paths. Additionally, the increase of $S_{e}$ and $S_{f}$ values confirms that fibers are better utilized with more candidate paths. However, for values larger than 5, additional paths do not further improve the number of required migrated components. Additional paths, up to some extent, allow for a better demand allocation using longer paths applying less efficient MFs, but using already spatially enabled fibers. This is confirmed in Fig. 5, which presents the percentage usage of all MFs as a function of the number of candidate paths. As seen, the higher the number of candidate paths, the higher the amount of demands using BPSK and the lower the amount of demands using more spectrally efficient MFs, such as QPSK or 8-QAM.

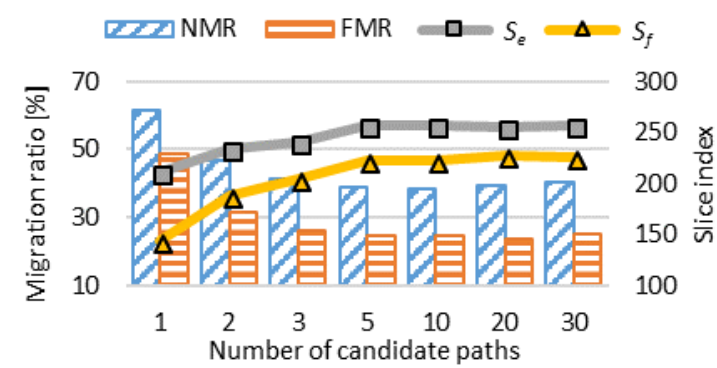

Fig. 4. NMR and FMR as a function of the number of candidate paths. 


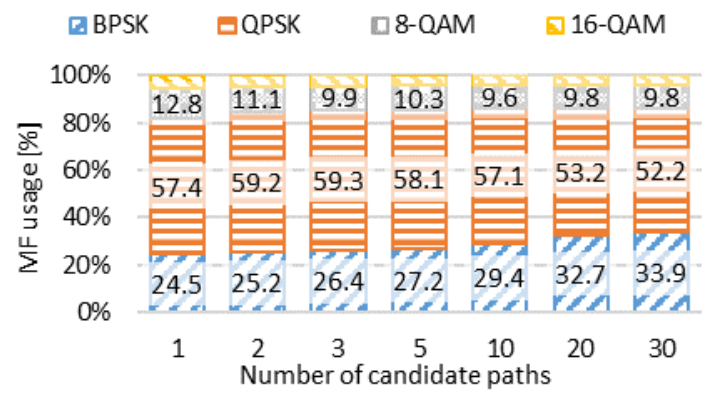

Fig. 5. MF usage as a function of number of the candidate paths.

Finally, we investigate the impact of the number of fibers $C$ within SMFBs. Note that lowering the active number of fibers in SMFBs result in a complexity reduction of SDMROADMs. In Fig. 6, NMR and FMR are presented as a function of number of fibers in SMFB links for traffic of 100 Tbps. Performance differences are observed only between 3 and 5 fibers, $16 \%$ and $22 \%$ for NMR and FMR, respectively. Further, fibers are underutilized, which confirms the decreasing value of $S_{f}$. In turn, the same function for 200 Tbps traffic is presented in Fig. 7. Here, NMR and FMR decrease by around $26 \%$ and $49 \%$ when moving from 3 to 11 fibers, respectively.

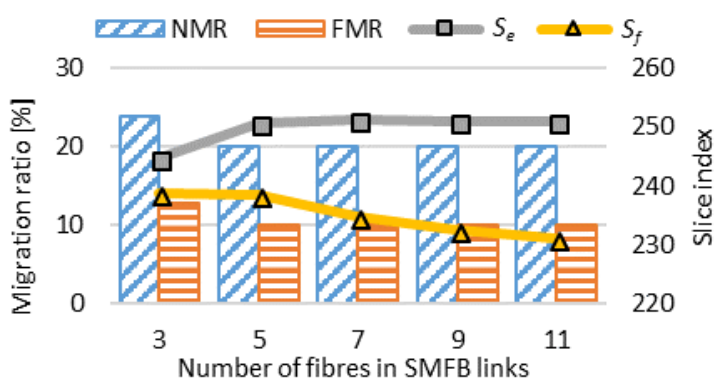

Fig. 6. NMR and FMR as a function of number of fibers in SMFBs, for a total traffic of $100 \mathrm{Tbps}$.

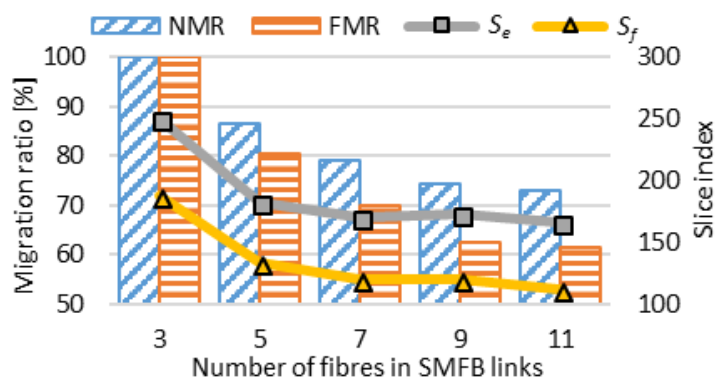

Fig. 7. NMR and FMR as a function of the number of fibers in SMFBs, for a total traffic of 200 Tbps.

\section{CONCLUSIONS}

We evaluated the performance of several nodes selection strategies in terms of gradual migration from EONs to SSFONs. According to the results, LCS strategy outperforms other proposed ones. In particular, by migrating $30 \%$ of regular ROADMs, a 2 times carried traffic increase can be achieved. Moreover, the impact of number of candidate paths and number of spatial channels on the required migration ratio is significant. As a future work, we plan to develop Simulated Annealing based node selection strategy for EONs to SS-FONs migration.

\section{ACKNOWLEDGMENT}

The work of K. Walkowiak and P. Lechowicz was supported by National Science Centre, Poland under Grant 2015/19/B/ST7/02490. R. Rumipamba-Zambrano is sponsored by SENESCYT - Ecuador.

\section{REFERENCES}

[1] I. Tomkos, B. Shariati, J. M. Rivas-Moscoso, D. M. Marom and D. Klonidis, "New frontiers in optical communication networking," 2017 19th International Conference on Transparent Optical Networks (ICTON), Girona, 2017, pp. 1-2.

[2] M. Klinkowski, P. Lechowicz, and K. Walkowiak, „Survey of resource allocation schemes and algorithms in spectrally-spatially flexible optical networking," Optical Switching and Networking, vol. 27 , pp. 58-78, 2018.

[3] R. Rumipamba-Zambrano, F. J. Moreno-Muro, P. Pavón-Marino, J. Perelló, S. Spadaro and J. Solé-Pareta, "Assessment of flex-grid/MCF optical networks with ROADM limited core switching capability," 2017 International Conference on Optical Network Design and Modeling (ONDM), Budapest, 2017, pp. 1-6.

[4] P. S. Khodashenas et al., "Comparison of spectral and spatial superchannel allocation schemes for SDM networks," Journal of Lightwave Technology, vol. 34, no. 11, pp. 2710-2716, 2016.

[5] C. Rottondi, P. Boffi, P. Martelli and M. Tornatore, "Routing, modulation format, baud rate and spectrum allocation in optical metro rings with flexible grid and few-mode transmission," in Journal of Lightwave Technology, vol. 35, no. 1, pp. 61-70, 2017. 